\title{
Resource Management in a New Green-IT World
}

\author{
Mauro Biagi \\ Department of Information, Electronics and Telecommunications engineering (DIET) \\ Sapienza, University of Rome
}

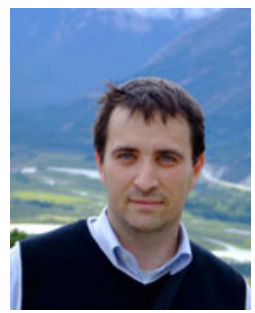

Wireless mobile, cabled xDSL, Fiber Optical Systems and Power Line Communications share a common problem: Bandwidth-Power efficiencies. While the efficiency required for the spectrum usage is different from wireless to wireline since the former has to respect some standardization bodies recommendations and the latter is mainly tied to channel quality aspects, the power efficiency is mainly linked to electrical power that comes from different sources like carbon, gas, oil, wind, solar, water. Unfortunately only few of these are renewable and require institutional investments for the medium-far future. In the meantime the only one way to be run appears to be the efficient. From this the necessity, in a Green-IT vision, to propose schemes, architectures, protocols and algorithms able to optimally manage bandwidth and power so to guarantee the expected performance at a reasonable cost. This is the direction currently followed by scientific community for already existing systems and in-developing new technologies.

Hence, each optimizing procedure with constraints on power/bandwidth usage or with the goal of power minimization or spectrum allocation, falls in the framework of a smart management of resources, so bit-loading for multi-carrier systems, coding and access techniques, scheduling and diversity schemes can be interpreted as an instance of Green-IT. In this context also the dualism between convex (closed-form) optimization and recursive algorithm takes place since also the complexity of the algorithms involves a power aspect, the power used for processing. This keynote, tries to show different approaches to efficiency by highlighting strong aspects and drawbacks for the present and next future. 